\title{
Inkjet Printing Ceramics: From Drops to Solid
}

\author{
B. Derby
}

School of Materials, University of Manchester,

Oxford Road, Manchester, M13 9PL, UK.

Inkjet printing is a powerful microfabrication tool that has been applied to the manufacture of ceramic components. To successfully fabricate ceramic objects a number of conditions must be satisfied concerning fluid properties and drop placement accuracy. It has been proposed that fluids are printable within the bounds $1<Z<10$ (where $Z$ is the inverse of the Ohnesorge number) and these limits are shown to be consistent with ceramic suspensions delivered by piezoelectric drop-ondemand inkjet printers. The physical processes that occur during drop impact and spreading are reviewed and these are shown to define the minimum feature size attainable for a given printed drop diameter. Finally the defects that can occur during the drying of printed drops are reviewed (coffee staining) and mechanisms and methodologies to reduce this phenomenon are discussed. 


\section{Introduction}

Inkjet printing has major commercial applications in graphics output and other conventional printing operations. However, there has been developing interest in using inkjet printing to manufacture components with applications for: displays ${ }^{1}$, plastic electronics ${ }^{2}$, rapid prototyping ${ }^{3}$, tissue engineering ${ }^{4}$, and ceramic component manufacture ${ }^{5}$. A significant and fundamental difference between these new applications and the more widespread application of printing text or images is the behaiour of the printed ink droplets on the printed substrate. Most images are constructed by the deposition of discrete droplets and, in order to optimise resolution and contrast, these droplets are isolated and do not contact each other. In contrast, many of the new applications for inkjet printing envisage the manufacture of continuous 1-, 2-, or 3-dimensional structures (1-, 2-, or 3-D). Such structures require a continuous distribution of material and this necessitates contact and adhesion between individual drops after printing.

Inkjet printing constructs objects by the precision placement of picolitre volumes of liquid and thus the initial interaction between printed material and a substrate is a liquid/solid interaction. Ultimately, the printed deposit undergoes a solidification process that can occur through solvent evaporation, temperature induced solidification/gelation or chemical reaction. Considerations of the relative timescales of drop spreading and solidification indicate that there will be a significant period of time after printing when a liquid is present on a surface ${ }^{6}$ and thus the morphological stability of coallescing liquid films must be examined, as must the effects of the solidification process. 
There has been a considerable number of publications on the use of inkjet printing in the manufacture of ceramics. ${ }^{7-17}$ These prior studies have used all inkjet drop generation technologies (continuous, thermal drop-on-demand and piezoelectric dropon-demand) to successfully produce ceramic objects using both solvent evaporation and phase-change solidification. Industrial inkjet printing technology now uses piezoelectric drop-on-demand (DOD) generation technology and this is the chosen method for most applications in printing functional materials. The physical operation of these different printing technologies and the reasons for the choice of piezoelectric DOD printing have been discussed in detail elsewhere, ${ }^{6,18}$ hence here we will confine our considerations to this technology. We will also only consider the printing of ceramic inks that solidify through solvent evaporation. Despite earlier work demonstrating that it is possible to successfully print $\mathrm{cm}$ scale objects using a wax based phase change ceramic ink, ${ }^{11-13}$ ceramic inks contain relatively low volume fractions of solid and thus there is considerable shrinkage and potential for distortion during dewaxing and sintering. ${ }^{13}$

In order to fabricate ceramic objects using inkjet printing, it is necessary to satisfy a number of requirements. First there is a need to produce stable ceramic suspensions with defined fluid properties such that they can be passed through a droplet generator and form regular drops. Second, these suspensions need to be delivered onto a substrate or onto a previously printed layer of solidified ceramic ink, with drops in sufficient proximity to each other to allow them to interact and form desired 2-D features. Next, the printed ceramic ink must undergo phase transition to a solid deposit. Finally, to produce 3-D structures the deposition and drying processes need to 
be repeated on a layer of pre-deposited and dried material. Here we will consider each of these requirements and their optimization for the direct printing of ceramics.

\section{Ceramic Inks}

Manufacturers of DOD inkjet printing equipment normally state a range of viscosity and surface tension within which inks may be successfully printed. However, this information is normally provided for the benefit of formulating graphics inks and may not be directly applicable to the development of ceramic inks. This is because inks containing a significant volume fraction of ceramic particles in suspension have much higher density values than typical graphics inks, which typically have densities in the range $800-1000 \mathrm{kgm}^{-3}$ and the behaviour of a fluid during printing depends strongly on its inertial behaviour.

The fluid rheological requirements for a printable ink are determined by the physics and fluid mechanics of the drop generation process. ${ }^{6,18}$ The behaviour of fluids during inkjet printing can be represented by the Reynolds, Weber and Ohnesorge numbers $(\operatorname{Re}, \mathrm{We}, \mathrm{Oh})$ :

$$
\begin{gathered}
\operatorname{Re}=\frac{v \rho a}{\eta} \\
W e=\frac{v^{2} \rho a}{\gamma} \\
O h=\frac{\sqrt{W e}}{\operatorname{Re}}=\frac{\eta}{(\gamma \rho a)^{1 / 2}}
\end{gathered}
$$


where $\rho, \eta$ and $\gamma$ are the density, dynamic viscosity and surface tension of the fluid respectively, $v$ is the velocity and $a$ is a characteristic length.

Fromm identified the Ohnesorge number, $\mathrm{Oh}$, as the appropriate grouping of physical constants to characterise drop generation in an inkjet printer. ${ }^{19} \mathrm{Oh}$ is independent of fluid velocity and is commonly used in analyses describing the behaviour of liquid drops. However, in Fromm's publication, he defined the parameter $Z=1 / O h$ and from a simple model of fluid flow in a drop generator of simplified geometry, he proposed that $Z>2$ for stable drop generation. ${ }^{19}$ Reis extended this through numerical simulation and proposed the following range, $10>Z>1$, for stable drop formation. ${ }^{20}$ If $Z<1$, viscous dissipation prevents drop ejection from the printer and if $Z>10$, droplets are accompanied by unwanted satellite drops. Jang et al studied the DOD printability of a number of fluid mixtures of ethanol, water and ethylene glycol. Through this they explored a range of values of $O h$ and determined that the range of printability was $4<\mathrm{Z}<14,{ }^{21}$ which is very similar to that determined by Reis's numerical simulation.

There is now a substantial body of literature describing the inkjet printing of a number of ceramic suspensions and other fluids for non-graphics applications; unfortunately not all publications report sufficient information on the rheological properties of the ceramic suspensions to test this proposed criterion for printability in all cases. Figure 1 presents such data that either reported the value of $O h$ (or $Z$ ) or reported sufficient data that it is easily calculated. The vertical dashed lines on the figure at $O h=1$ and $O h=10$ represent the limits for stable inkjet printing calculated by Reis. ${ }^{20}$ The 
experimental data is presented from 8 fluid systems with a grey symbol indicating the successful printing of individual drops, a black symbol indicates that fluids with these properties could not be printed, and finally a white symbol shows the cases where a fluid drop was successfully ejected but accompanied by one or more satellite drops. It is useful to separate these data into two sets: fluid systems $1-6$ were delivered using piezoelectric DOD printers, while fluid systems 7 and 8 were delivered using a thermal DOD printer. The data obtained from experiments using piezoelectric DOD printing shows reasonably good agreement with Reis's model, however that obtained in the one study using a thermal DOD printer shows very poor agreement, ${ }^{17}$ at least with the upper bound for the prediction of the onset of satellite drop formation. Özkol considered that one reason for the discrepancy between Reis's prediction and their results could be the difference in actuation between piezoelectric and thermal DOD inkjet droplet generators. ${ }^{17}$

The hypothesis that changes in actuation explain the different behaviour observed between thermal and piezoelectric DOD inkjet printing is supported by an experimental study of drop and satellite formation in a piezoelectric DOD printer by Dong et al. ${ }^{22}$ They found that the drop formation mechanism and the conditions under which a given fluid formed satellites is also controlled by the shape and amplitude of the driving pulse applied to the piezoelectric actuator. The driving pulse in DOD printing is also known to control both the size of the ejected drop and its velocity. ${ }^{12,22,23}$ Reis demonstrated that for the formation of drops using highly loaded ceramic suspensions, acoustic phenomena are important and that there are maxima in inkjet performance that correlate with acoustic resonances in the printhead. ${ }^{23}$ These are particularly important considerations given that typical industrial DOD printheads 
operate in the $\mathrm{kHz}$ regime. Other studies of inkjet printing for applications in graphics also emphasise the importance of acoustic phenomena and the need for these to damp before the drop generator is refilled prior to delivering subsequent drops. ${ }^{18}$ Indeed the shape and form of the actuating waveform is considered an important aspect of the design of piezoelectric DOD printing systems.

However, from figure 1, we can see that for the studies that used piezoelectric DOD printers, Reis's criterion for a printable fluid ${ }^{20}$ seems to show reasonable agreement with data and it is also in broad agreement with the only explicit study of inkjet printability of fluids by Jang et al. ${ }^{21}$ Thus despite a possible oversimplification of the conditions that lead to the formation of satellite drops, we suggest the condition $10>$ $Z>1$ (where $Z=1 / O h$ ) can be used as a guide to the development of fluids for ink jet printing.

The suitability of a fluid for inkjet printing can be roughly assessed by its Ohnesorge number. However there are other limits of fluid behaviour that impose additional limits to practical drop generation. In order to generate a small radius drop, the surface tension and associated Laplace pressure must be overcome before a drop can be ejected from a printer. Duineveld proposed that this can be described by a minimum value of the Weber number, $W e>4$, below which there is insufficient fluid flow to overcome surface tension. ${ }^{24} \mathrm{~A}$ final bound to printability is given by the onset of splashing that occurs if a drop hits the substrate with velocity above a critical threshold. From the work of Stowe and Hadfield, ${ }^{25}$ this occurs when $W e^{1 / 2} R e^{1 / 4}>50$. These limiting bounds define a region of the parameter space of $W e$ and $R e$, within which DOD inkjet printing is possible. ${ }^{5,6}$ Figure 2 shows this parameter space and the 
region suitable for DOD inkjet printing. Drop velocity increases diagonally, as indicated and has lower and upper bounds that are defined by the appropriate limits of drop ejection and splashing, orthogonal to velocity is the Ohnesorge number, which defines the limits of the fluid properties, thus figure 2 can be considered representing a guide to the limits of both fluid characteristics and drop dynamics consistent with the practical use of piezoelectric DOD inkjet printing.

\section{Drop Impact, Spreading and Coalescence}

As discussed earlier, an important aspect of inkjet printing in manufacturing technology is the process by which adjacent drops interact to form a solid. In all cases the liquid drop will interact with a solid substrate. Following deposition there will be a period when the drop's shape is controlled by fluid processes prior to solidification. Thus an important consideration is the appropriate time constants that apply to the mechanisms of surface spreading and solidification. Here we are confining our discussion to solidification through evaporation. Given that droplet solidification time scales are normally in the regime of around $1 \mathrm{~s}$ and droplet deposition rates are $>1$ $\mathrm{kHz}$, we need to consider the interaction between many liquid droplets on the surface of the substrate. It is possible to use interlacing and sequential printing passes to deposit isolated drops, allow them to solidify and then fill in the gaps to produce a printed plane. However, this methodology produces an irregular deposit with poor surface roughness for each printed layer, ${ }^{9}$ with a consequent risk of defects from poor penetration of the liquid. If printing occurs with appropriate drop spacing to allow overlap before solidification, the interaction between adjacent liquid drops and the 
consequent influence of surface tension will tend to produce smooth surfaces and eliminate possible defects between solidified drops.

When a liquid drop impacts a planar substrate it will deform and spread to cover the substrate, ultimately achieving an equilibrium sessile drop configuration. Yarin has recently reviewed the impact of drops over a size and velocity range that intersects those relevant to DOD printing. ${ }^{26}$ The typical range of drop size (radius from 5 - 50 $\mu \mathrm{m})$ and velocity $\left(1<v<10 \mathrm{~ms}^{-1}\right)$ is such that the initial deformation of the drop will be controlled by dynamic impact and viscous dissipation processes. ${ }^{6,18,26}$ However, this initial stage of drop deformation is expected to have finished after a few $\mu$ s and subsequent spreading to equilibrium will be driven by capillary forces. ${ }^{27} \mathrm{~A}$ schematic representation of the timescales associated with drop deformation after impact is presented in figure 3. The dynamic processes of drop impact occur over a time scale of $\mu \mathrm{s} .{ }^{26}$ First the drop deforms on impact with its kinetic energy converted into new surface area as the drop deforms, some energy is absorbed through viscous dissipation. If the impact conditions are such that splashing does not occur (as is normal with the conditions of inkjet printing), the drop may recoil after expansion and oscillate briefly dissipating energy. Meanwhile the process of capillary spreading will occur, this has a time scale of ms for drop dimensions consistent with inkjet printing and the final equilibrium drop shape is normally controlled by this process.

Once equilibrium has been reached, the drop can be modelled as a spherical cap because the Bond number is sufficiently low that we may ignore the influence of gravity. In which case the equilibrium contact diameter of the drop, $d_{\text {eqm }}$, can be calculated with 


$$
d_{\text {eqm }}=\beta d_{0}
$$

where

$$
\beta=2\left\{\tan \frac{\theta_{\text {eqm }}}{2}\left(3+\tan ^{2} \frac{\theta_{\text {eqm }}}{2}\right)\right\}^{-1 / 3}
$$

where $d_{0}$ is the diameter of the drop in flight and $\theta_{\text {eqm }}$ is the equilibrium contact angle. For an isolated drop of pure solvent, we would expect the drop diameter to decrease and the contact line to retract at a constant receding contact angle during evaporation. However, for the case of particles in suspension, the behaviour of a liquid drop is different. Solvent evaporation does not occur uniformly from the sessile drop. At low contact angles, the fluid close to the contact line is adjacent to a large dry surface and this enhances the transport of the solvent vapour promoting faster evaporation. This leads to a ring of particles coming out of suspension and the presence of this dried deposit pins the contact line and prevents it retracting. This contact line pinning results in the receding contact angle decreasing as solvent is removed. It can also result in a flow of particles to the contact line, leading to suspension segregation and a ring deposit; this is a phenomenon known as the coffee stain effect. ${ }^{28}$ We will return to the coffee stain phenomenon later in this article. One effect of contact line pinning during drying is that the footprint or equilibrium diameter of the spread drop of ceramic ink will define the diameter of the dried deposit on the surface after solvent evaporation.

In order to print 2-dimensional patterns it is necessary to allow adjacent droplets to interact and coalesce. It is advantageous for these drops to interact while in a liquid state because surface tension forces will result in a smooth deposit surface. If we 
consider the interaction of adjacent liquid drops in the absence of contact line pinning, two drops on merging would tend to form a large spherical cap to minimise surface area. However, if contact lines are pinned fluid flow is limited and a train of interacting drops will form a linear feature. This was formalised by Davis who demonstrated that a liquid bead was stable against breaking up into isolated spherical caps if the receding contact angle was free to change but the contact line was pinned ${ }^{29}$ this was confirmed experimentally by Schiaffino and Sonin. ${ }^{30}$ Given this assumption of line stability it is possible to compute the width, $w$, of a stable liquid bead formed by the overlap of a train of drops of diameter $d_{0}$ and spacing $p$ (figure 4), with: ${ }^{31}$

$$
w=\sqrt{\frac{2 \pi d_{0}^{3}}{3 p\left(\frac{\theta_{e q m}}{\sin ^{2} \theta_{e q m}}-\frac{\cos \theta_{e q m}}{\sin \theta_{e q m}}\right)}}
$$

Clearly if $p>d_{\text {eqm }}$ no continuous track or liquid bead is formed. Stringer developed this expression further to show that because the receding contact line is pinned, equation 3 is only valid if $w>d_{\text {eqm. }}{ }^{32}$ If the drop spacing is such that $w<d_{\text {eqm }}$, the individual drops have to recede to form a parallel sided track but because the contact line is pinned (the condition for stability of a liquid bead), the resulting liquid track has non-parallel sides (figure $5 b$ ). Thus the maximum spacing of drops, $p_{\max }$, to produce a parallel sided liquid bead can be obtained by inserting $w=p_{\max }$ into equation 3 and solving to give 


$$
p_{\text {max }}=\frac{2 \pi d_{0}}{3 \beta^{2}\left(\frac{\theta_{\text {eqm }}}{\sin ^{2} \theta_{\text {eqm }}}-\frac{\cos \theta_{\text {eqm }}}{\sin \theta_{\text {eqm }}}\right)}
$$

Figure 5, taken from the publication of Soltman and Subramanian, ${ }^{33}$ shows the behaviour of inkjet printed tracks as the drop spacing reduces. In figure 5a, $p>d_{\text {eqm }}$, resulting in isolated and separated drops; while in figure $5 \mathrm{c}, p<p_{\max }$ and a parallel sided track is formed. Figure $5 \mathrm{~b}$ shows the intermediate state where the pinned contact line results in an irregular contact line bounding the track. It should be noted that figure $5 \mathrm{~d}$ shows that, as the drop spacing reduces further, another limiting value of drop spacing is encountered. When the drop spacing reduces below some minimum value, the track width no longer increases uniformly but now a series of bulges appear along the previously parallel sided track. This bulge instability was first investigated in detail by Duineveld, who modelled its features as the result of a dynamic instability that occurs because of competing flow paths for a newly arrived drop. ${ }^{34}$ When a newly arrived drop begins to spread across the substrate and intersects the pre-existing liquid bead, fluid flow can either drive the spreading or else flow down the bead. Duineveld demonstrated that at low drop centre spacing and when the drops arrived at relatively long time intervals, flow down the liquid bead was preferred. Conversely at larger droplet spacing and rapid drop arrival rates, spreading flow was favoured. Bulging occurs because flow down the liquid bead leads to an increase in the local contact angle removing one of the constraints that induces stability, proposed by Davis. ${ }^{29}$ Thus this bulging instability is dynamic and the threshold for its onset is a function of both drop spacing and the rate of arrival of drops, which is the velocity at which the printhead traverses the substrate, $U_{T}$, divided by the drop spacing. 
Stringer adapted Duineveld's model to obtain an analytical expression for the onset of the bulging instability, ${ }^{32}$ which can be expressed in terms of a dimensionless traverse velocity, $U_{T}{ }^{*}$, falling below a function of the advancing contact angle, $\theta_{a d v}$ and a dimensionless drop spacing, $g\left(p^{*}, \theta\right)$. Thus the condition for a stable line is given by

$$
\begin{array}{r}
U_{T}^{*}>g\left(p^{*}, \theta_{a d v}\right) \\
\text { with } \quad U_{T}^{*}=\frac{U_{T} \eta}{\gamma}
\end{array}
$$

The function $g\left(p^{*}, \theta\right)$ is related to the inverse of the drop spacing and the contact angle and is given explicitly as equation 16c in ref. [33]. Figure 6 shows Stringer's formulation of Duineveld's instability model, superimposed upon which is the data for three different fluids on a range of substrates including: Ag nanoparticle ink, ${ }^{33}$ polymer solution (PEDOT/PSS), ${ }^{34}$ and a $\mathrm{ZrO}_{2}$ ceramic suspension. ${ }^{35}$ With the exception of one set of experiments from Duineveld's initial study, the experimental data shows excellent agreement with Stringer's predictions. The data that does not fit the model comes from a fluid/substrate system with a very low advancing contact angle and there is evidence that Duineveld's model may not be applicable in such cases; this is discussed in more detail elsewhere. ${ }^{32,34,36}$ However, when the advancing contact angle is very low the minimum feature dimension (as defined by equation 3 ) will be very large and such fluid substrate combinations are unlikely for practical manufacturing applications. 
It is possible to combine the expressions for the two limiting bounds for droplet spacing by determining an appropriate value for $g\left(p^{*}, \theta_{a}\right)$ at the value of $p^{*}$ that describes the maximum allowable droplet spacing for parallel track formation, $g\left(p^{*}{ }_{\max }, \theta_{a}\right)$, and plotting this on the same set of axes used to define the onset of the bulge instability in figure $6 .^{32}$ This is shown in figure 7 where a triangular region of stability is shown in a parameter space defined by axes of $g\left(p^{*}{ }_{\max }, \theta_{a}\right)$ and $U^{*}$. The diagonal line is the limiting case for the bulge instability as set out in figure 7 , the vertical line represents the location of $p^{*}$, the dimensionless drop spacing, but as $g\left(p^{*}, \theta_{a}\right)$ include the advancing contact angle, the position of $p^{*}{ }_{\max }$ moves for different values of the contact angle. The horizontal bounding line indicates that for any printing equipment, there is a limit to the maximum traverse velocity for an inkjet printhead. This diagram can be used to identify the stable region of drop spacing usable when inkjet printing lines and by inference the printing of sheets of material by overlapping lines. The conditions for stable drop spacing are a strong function of contact angle, with the region of stability decreasing with increasing contact angle. Thus by increasing the contact angle to reduce the minimum feature size, we restrict the stable range of drop spacing.

\section{Drop Drying and Coffee Staining}

The final process to consider that is important in controlling deposit shape is drop drying and coffee staining. Deegan explained this phenomenon as being caused by a combination of contact line pinning preventing an evaporating drop receding, as volume is lost through evaporation, and the enhanced evaporation that occurs at the bounding contact line of a well spread drop, which is driven by the large region of dry 
substrate surrounding the drop that enhances diffusion of the evaporating vapour from the drop surface. ${ }^{28}$ In a later publication Deegan et al presented a detailed study of coffee staining and developed a numerical model for the process that agreed well with experiment. $^{37}$ They concluded that coffee staining is the normal behaviour for a drying drop over all length scales studied for both solute and suspended particles. It is not caused by the surrounding dry surface, as originally proposed, ${ }^{28}$ but occurs if the following two conditions are obeyed: 1 ) the edge of the drying drop (the contact line) is pinned and 2) evaporation is possible from the edge of the drop. For solutions or suspensions, condition 1 is normally met if there is any significant roughness to the surface and at small contact angles when rapid solid deposition occurs at the contact line. Deegan observed that on smooth PTFE substrates contact line pinning was absent and drops contracted during evaporation to leave an uniform deposit. ${ }^{37}$ Deegan also explored constraining evaporation through a hole positioned above a drying drop, which severely reduces evaporation from the edge of the drop, and this to eliminated coffee staining.

Coffee staining has been observed in a number of prior studies using inkjet printing and methods to counter its effects have been proposed and studied. First we note that coffee staining is only a problem when the liquid solidifies through evaporation and is not significant when a liquid drop solidifies through a phase change on impact. ${ }^{14,38}$ However, as discussed earlier, the use of an evaporating solvent is advantageous in printing ceramics to ensure maximum solid density prior to sintering. Deegan noted that it may be possible to counter the fluid flow caused by differential evaporation through inducing an opposing flow from the Marangoni effect. ${ }^{37}$ He noted that the main source of heat to drive evaporation arises from contact with the substrate, hence 
the centre of the drop is expected to be cooler than the edge. In most fluids surface tension decreases with increasing temperature thus there is expected to be a gradient in surface tension that increases to a maximum at the drop centre. Deegan did observe a flow opposing coffee staining during evaporation but this decreases with time and is insufficient to prevent coffee staining. De Gans and Schubert exploited the Marangoni effect through the use of solvent mixtures. ${ }^{39}$ Two solvents were selected, with different vapour pressure and surface tension values, such that a high vapour pressure solvent evaporated at the drop edge causing a local decrease in surface tension and thus generated a surface tension gradient increasing towards the drop centre. Such solvent mixtures can generate much larger surface tension gradients than are attainable through temperature gradients and they were able to completely suppress coffee staining following inkjet printing of polystyrene solutions. The use of solvent mixtures to suppress coffee staining was also shown to be applicable to ceramic suspensions by Zhao et al. ${ }^{40}$ A number of other parameters have been shown to influence coffee staining including the spacing of droplets, ${ }^{39}$ local partial pressure of the solvent, ${ }^{40}$ and even the impact velocity of the drops. ${ }^{16}$

The importance of Marangoni flows during droplet drying can be estimated using the dimensionless Marangoni number, $M a$, defined:

$$
M a=\frac{\Delta \gamma r}{\eta D}
$$

Here, $\Delta \gamma$ is the difference in surface tension (taken here to be the difference between the two pure solvents), $r$ is a characteristic length (assumed to be the radius of the 
spread drop on the substrate), $\eta$ is the fluid viscosity and $D$ is the solute diffusion coefficient. It is generally believed that Marangoni flows are significant if $M a>100$. Both de Gans and Zhao computed very large values for $M a$ in their studies of approximately $10^{6} \mathrm{x} \Delta \gamma$ (using SI units). ${ }^{39,40}$ This result implies that even very small differences in surface tension of around $10^{-4} \mathrm{Jm}^{-2}$ should be sufficient to impede coffee staining and this would appear to be at odds with the common observation of coffee staining reported in the literature. The influence of Marangoni convection on coffee staining was investigated in considerable detail by Hu and Larson. ${ }^{41}$ They found that when clean organic solvents were used in drop drying experiments, coffee staining was never observed and that Marangoni flow dominated the evaporation driven flow proposed by Deegan. ${ }^{29}$ They further proposed that the suppression of Marangoni flow is a necessary condition for the observation of coffee staining. They suggested that the reason coffee staining is observed in most aqueous systems studied in the literature is because the Marangoni number is drastically reduced (Ma $<100)$ because of the strong influence of surface contaminants on the surface properties of water.

From this survey of recent work it is clear that solute or particle segregation during the drying of drops is an extremely complex process with a number of competing mechanisms in addition to evaporation and Marangoni flow. However, although we lack a full understanding of the mechanisms, it is evident that there are a number of possible routes available to develop formulations for ceramic suspensions that are printable and do not show coffee staining. 


\section{Conclusions}

Inkjet printing has been developed as a ceramic processing tool over the last 15 years and has been used as a tool in graphics output for over 30 years. Objects are fabricated by the interaction of individual drop deposited on a substrate or on previously deposited layers. The process is complex and involves a number of distinct steps that can be grouped into: 1) drop generation, 2) drop interaction with the substrate/deposit and adjacent drops, 3) the drying of drops to form a solid. None of these processes are fully understood but significant progress has been made to allow a rational approach to develop the technology as a ceramic processing tool. It is important to recognise the differences between inkjet printing as a ceramic (or general materials) processing tool and its use in graphics. Inkjet compatible ceramic inks tend to be significantly more viscous and denser than graphics inks and contain much larger volume fractions of solids in suspension. By far the majority of industrial inkjet equipment is designed for use with graphics inks and may not be easily transferred to printing ceramics. The interaction of droplets and the drop drying process are clearly important in determining the quality, accuracy and properties of a printed object. There has been little systematic work on these aspects of ceramic processing by inkjet printing in the literature, despite this, high quality ceramic components have been produced. Nonetheless much further work is needed to ensure that consistent objects can be produced using this technique for commercial applications. 


\section{References}

1. Bharathan J, Yang Y. Polymer electroluminescent devices processed by inkjet printing: I. Polymer light-emitting logo. Appl Phys Lett 1998;72:2660-2

2. Sirringhaus H, Kawase T, Friend RH, Shimoda T, Inbasekaran M, Wu W, et al. High-resolution inkjet printing of all-polymer transistor circuits. Science 2000;290:2123-6

3. Sachs E, Cima M, Williams P, Brancazio D, Cornie J. 3-Dimensional Printing - Rapid Tooling and Prototypes Directly from a CAD Model. J. Eng. Ind.Trans. ASME 1992;114:481-8

4. Nakamura M, Kobayashi A, Takagi F, Watanabe A, Hiruma Y, Ohuchi K, et al. Biocompatible inkjet printing technique for designed seeding of individual living cells. Tissue Eng. 2005;11:1658-66.

5. Derby B, Reis N. Inkjet printing of highly loaded particulate suspensions. MRS Bull. 2003;28:815-8.

6. Derby B. Inkjet printing of functional and structural materials - fluid property requirements, feature stability and resolution. Ann Rev Mater Res 2010;40:395-414.

7. Xiang QF, Evans JRG, Edirisinghe MJ, Blazdell PF. Solid freeforming of ceramics using a drop-on-demand jet printer, Proc Instn Mech Eng B J Eng Manuf 1997;211:211-4.

8. Slade CE, Evans JRG. Freeforming ceramics using a thermal jet printer, $J$ Mater Sci Lett 1998;17:1669-1671.

9. Mott M, Song JH, Evans JRG, Microengineering of ceramics by direct ink-jet printing, J Amer Ceram Soc, 1999:82:1653-8. 
10. Windle J, Derby B. Ink jet printing of PZT aqueous ceramic suspensions. $J$ Mater Sci Lett 1999;18:87-90.

11. Blazdell PF, Evans JRG. Application of a continuous ink jet printer to solid freeforming of ceramics, J Mater Process Tech 2000;99:94-102.

12. Seerden KAM, Reis N, Evans JRG, Grant PS, Halloran JW Derby B. Inkjet printing of wax based ceramic suspensions, J Amer Ceram Soc. 2001;84:251420.

13. Ainsley C, Reis N, Derby B. Freeform fabrication by controlled droplet deposition of powder-filled melts", J Mater Sci. 2002;37:3155-61.

14. Smith P, Derby B, Reis N, Wallwork A, Ainsley C, Measured anisotropy of alumina components produced by direct inkjet printing. Euro. Ceramics VIII, Pts. 1-3 Eds: H. Mandal and L. Ovecoglu, Key Eng. Mater. 2004;264-268:6936.

15. Wang T, Derby B. Inkjet printing and sintering of PZT. J Amer Ceram Soc 2005;88:2053-8.

16. Noguera R, Lejeune M, Chartier T. 3D fine scale ceramic components formed by ink-jet prototyping process. J. Europ. Ceram. Soc. 2005;25:2055-9.

17. Özkol, E, Ebert J, Telle R. An experimental analysis of the influence of the ink properties on the drop formation for direct thermal inkjet printing of high solid content aqueous 3Y-TZP suspensions. J. Europ. Ceram. Soc. 2010;30:166978.

18. Martin GD, Hoath SD, Hutchings IM. Inkjet printing - the physics of manipulating liquid jets and drops. J. Phys.: Conf. Ser. 2008;105:012001.

19. Fromm JE. Numerical-calculation of the fluid-dynamics of drop-on-demand jets. IBM J. Res. Dev. 1984;28:322-33. 
20. Reis N, Derby B. Ink jet deposition of ceramic suspensions: Modelling and experiments of droplet formation. Materials Development for Direct Write Technologies, Boston, MA, 2000, MRS Symp. Proc. 2000;624:65-70: Materials Res. Soc.

21. Jang D, Kim D, Moon J. Influence of fluid physical properties on ink-jet printability. Langmuir 2009;25:2629-35.

22. Dong H, Carr WC, Morris JF. An experimental study of drop-on-demand drop formation. Phys. Fluids 2006;18:072102.

23. Reis N, Ainsley C, Derby B. Inkjet delivery of particle suspensions by piezoelectric droplet ejectors. J. Appl. Phys. 2005;97:094903.

24. Duineveld PC, de Kok MA, Buechel M, Sempel AH, Mutsaers KAH, van de Weijer. P. Ink-jet printing of polymer light-emitting devices. Proc. Conference on Organic Light-Emitting Materials and Devices V, San Diego, Ca, 2001, 4464:59-67: SPIE-Int Soc Optical Engineering.

25. Stow CD, Hadfield MG. An experimental investigation of fluid-flow resulting from the impact of a water drop with an unyielding dry surface. Proc $R$ Soc Lon Ser A 1981;373:419-41.

26. Yarin AL. Drop impact dynamics: Splashing, spreading, receding, bouncing. Annu Rev Fluid Mech 2006;38:159-92.

27. Tanner LH. Spreading of silicone oil droplets on horizontal surfaces. J Phys D-Appl Phys 1979;12:1473-84.

28. Deegan RD, Bakajin O, Dupont TF, Huber G, Nagel SR, Witten TA. Capillary flow as the cause of ring stains from dried liquid drops. Nature 1997;389:8279. 
29. Davis SH. Moving contact lines and rivulet instabilities. 1. The static rivulet. $J$ Fluid Mech 1980;98:225-42.

30. Schiaffino S, Sonin AA. Formation and stability of liquid and molten beads on a solid surface. J Fluid Mech 1997;343:95-110.

31. Smith PJ, Shin DY, Stringer JE, Derby B, Reis N. Direct ink-jet printing and low temperature conversion of conductive silver patterns. J Mater Sci 2006;41:4153-8.

32. Stringer J, Derby B. Formation and stability of lines produced by inkjet printing. Langmuir 2010;26:10365-72.

33. Soltman D, Subramanian V. Inkjet-printed line morphologies and temperature control of the coffee ring effect. Langmuir 2008;24:2224-31.

34. Duineveld PC, The stability of ink-jet printed lines of liquid with zero receding contact angle on a homogeneous substrate. J. Fluid Mech. . 2003;477:175-200.

35. Dou R, Derby B. Inkjet Printing of Zirconia: Coffee Staining and Line Stability. J. Amer. Ceram. Soc. 2010:submitted.

36. Stringer J, Derby B. Limits to feature size and resolution in inkjet printing. $J$. Europ. Ceram. Soc. 2009;29:913-8.

37. Deegan RD, Bakajin O, Dupont TF, Huber G, Nagel SR, Witten TA. Contact line deposits in an evaporating drop. Phys. Rev. E 2000;62:756-65.

38. van den Berg AMJ, de Laat AWM, Smith PJ, Perelaer J, Schubert US. Geometric control of inkjet printed features using a gelating polymer. J. Mater. Chem. 2007;17:677-83.

39. de Gans BJ, Schubert US. Inkjet printing of well-defined polymer dots and arrays. Langmuir 2004;20:7789-93 
40. Zhang Y, Yang S, Chen L, Evans JRG. Shape changes during the drying of droplets of suspensions. Langmuir 2008;24:3752-8.

41. Hu H, Larson RG. Marangoni effect reverses coffee stain depositions. J. Phys. Chem. B 2006;110:7090-4. 


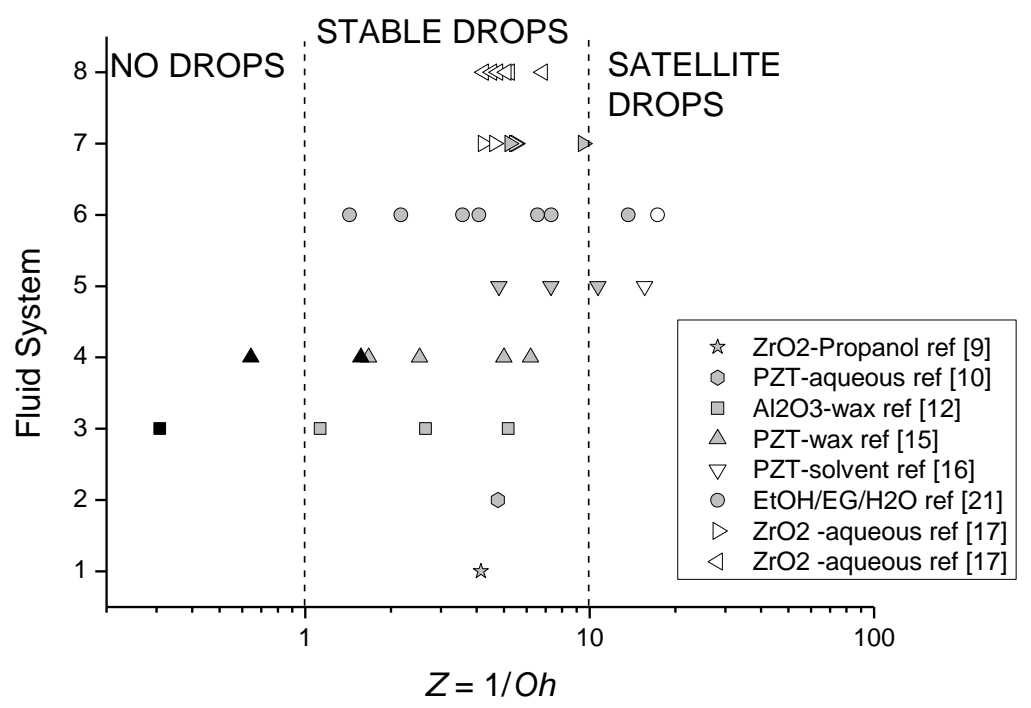

Figure 1 Fromm's parameter $Z(Z=1 / O h)$ influences the printability of fluids.

Dashed lines identify the limits for printability proposed by Reis et al. ${ }^{20}$ Experimental points are plotted for a number of ceramic suspensions/inks: grey symbols indicate successful inkjet printing, black symbols indicate that no drops were formed, and white symbols indicate the presence of satellite drops along with the main printed drop. 


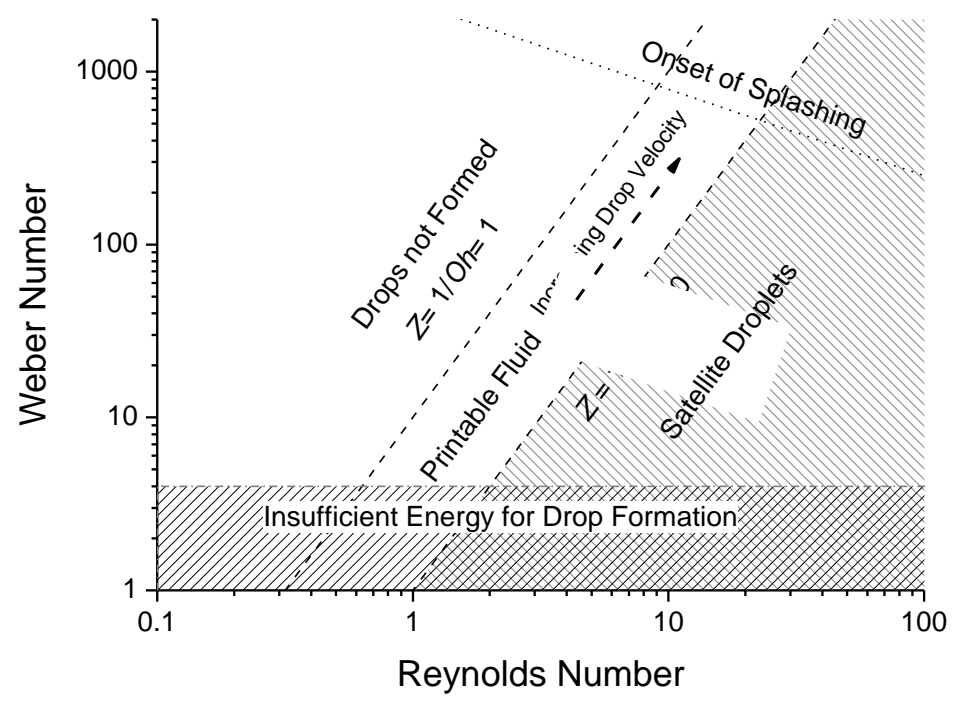

Figure 2 Inkjet printing is practical for a limited range of fluids and printing conditions. This is illustrated here in a parameter space defined by axes of Reynolds and Weber numbers. Based on a diagram originally published in ref. [5]. 


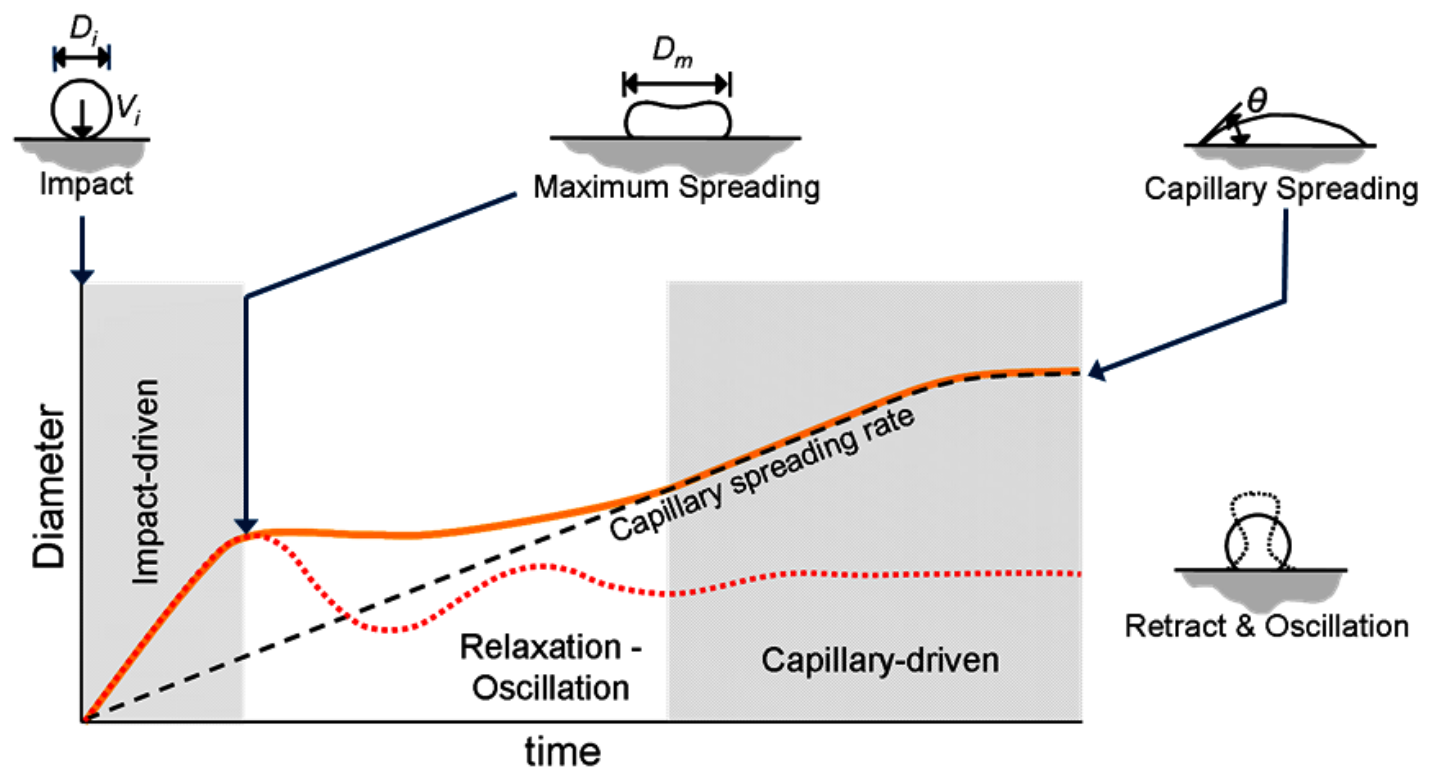

Figure 3 Schematic illustration of the time scales appropriate to the processes of drop deformation and spreading on a substrate after impact. Axes of diameter and time are to arbitrary non-linear scales. Reproduced with permission from reference [6]. 


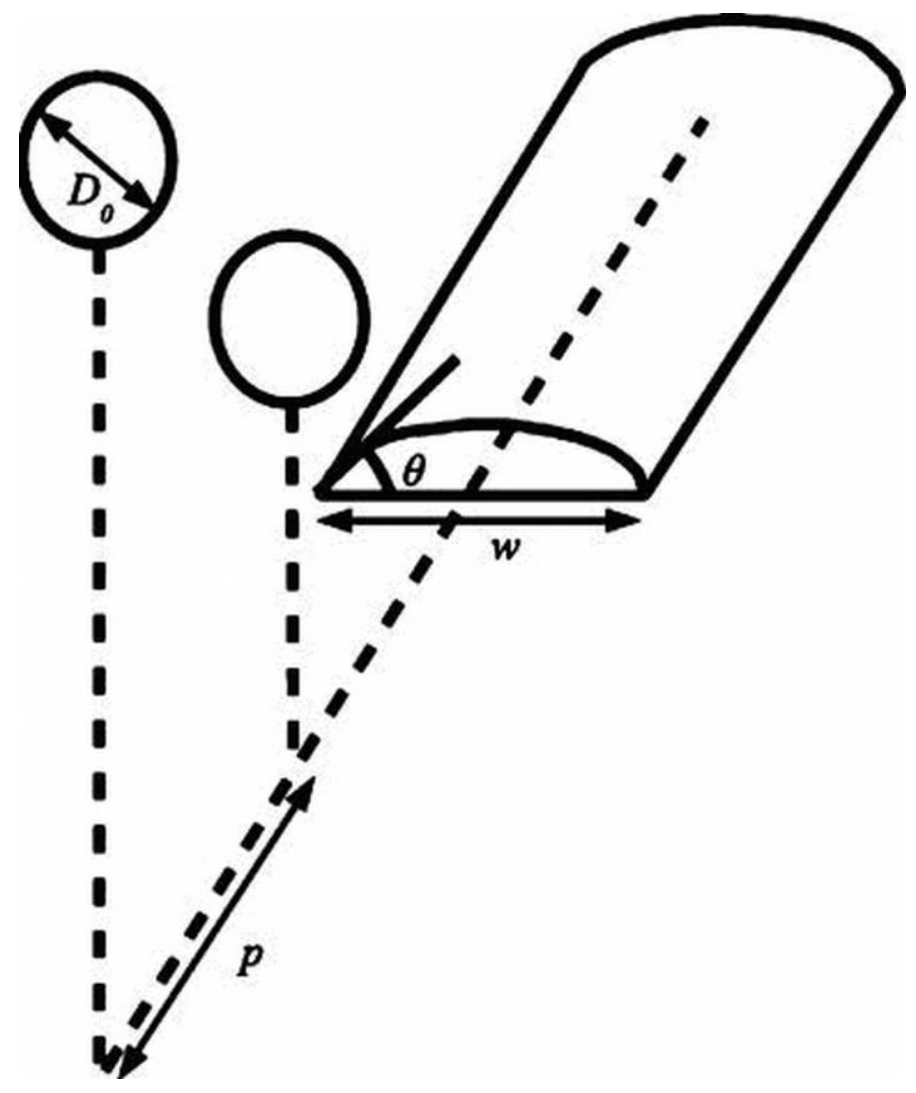

Figure 4 Schematic illustration of the coalescence of individual drops to form a track or liquid bead with a uniform cross section of a circular sector. Based on an illustration originally published in ref. [31]. 


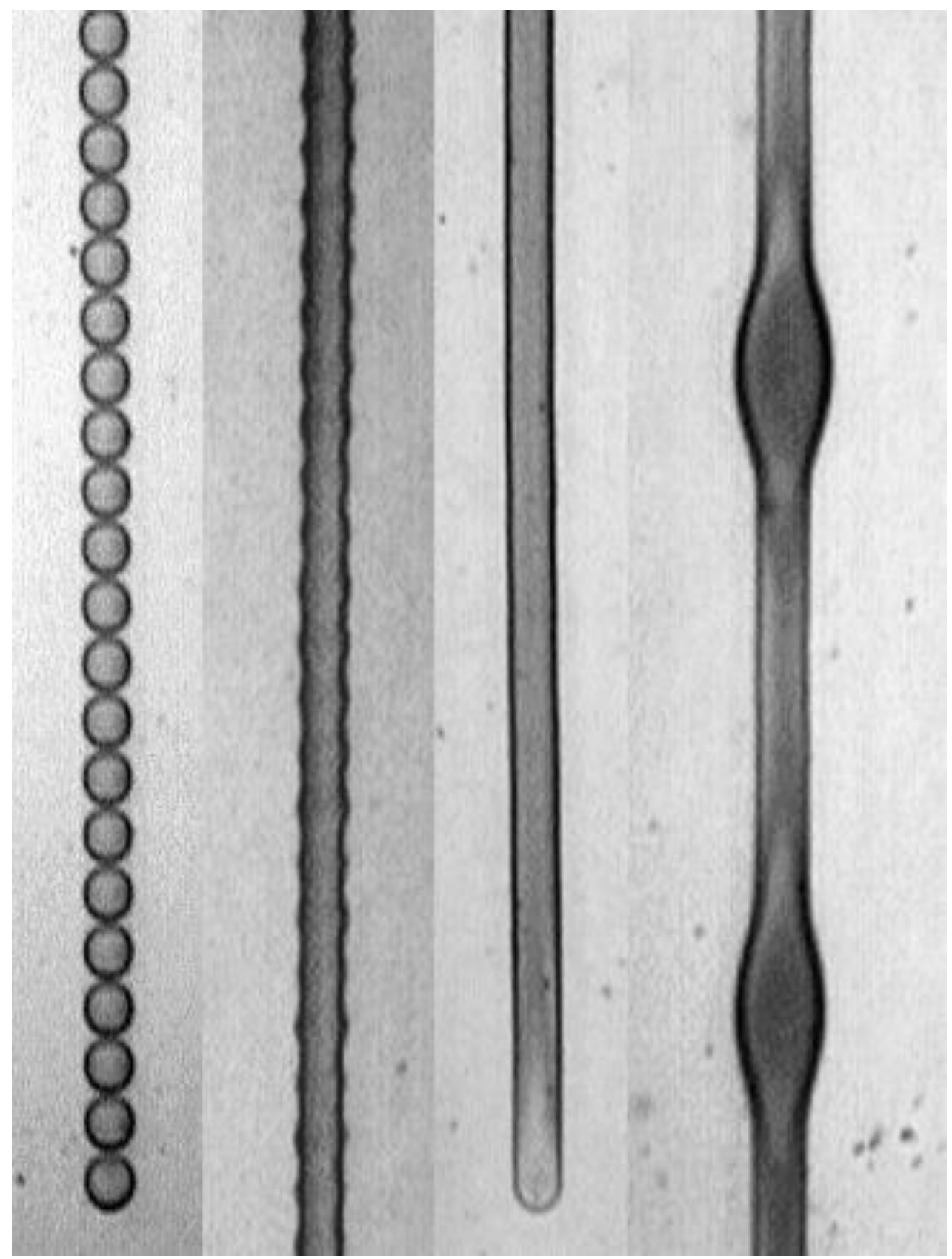

Figure 5 Four morphologies possible when individual drops are printed onto a surface at regularly spaced intervals: a) drops are spaced $p>d_{\text {eqm }}$ : no interaction occurs, b) $p_{\max }<p<d_{\text {eqm }}$ : a continuous track is formed but contact line pinning results in an irregular edge, c) $p<p_{\max }$ : parallel sided track is formed, d) when drop spacing is below a threshold determined by both contact angle and printing speed, a bulge instability develops. Reproduced with permission from ref. [33]. 


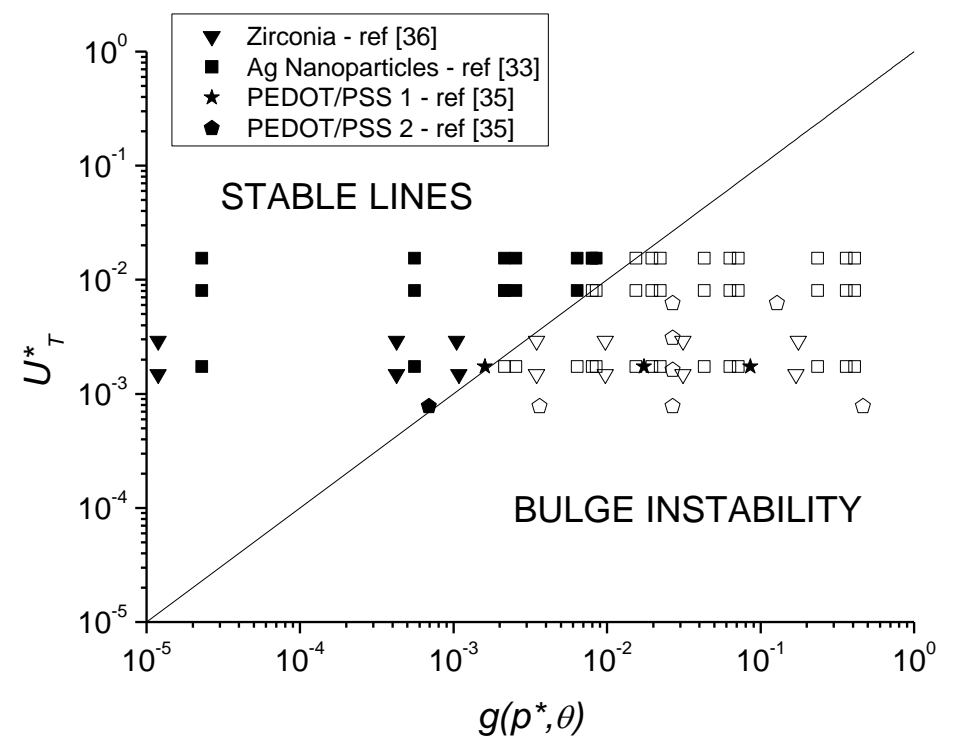

Figure 6 The bulge instability is observed below a critical drop spacing that depends on the traverse speed of the printer. Stringer's criterion for the onset of the instability in terms of the parameter $g\left(p^{*}, \theta\right)$ and a dimensionless velocity is shown as the solid line, ${ }^{32}$ with experimental data from a range of sources superimposed. Black symbols indicate well formed lines with parallel sides, white symbols indicate the conditions under which unstable bulges appear on printed lines. 


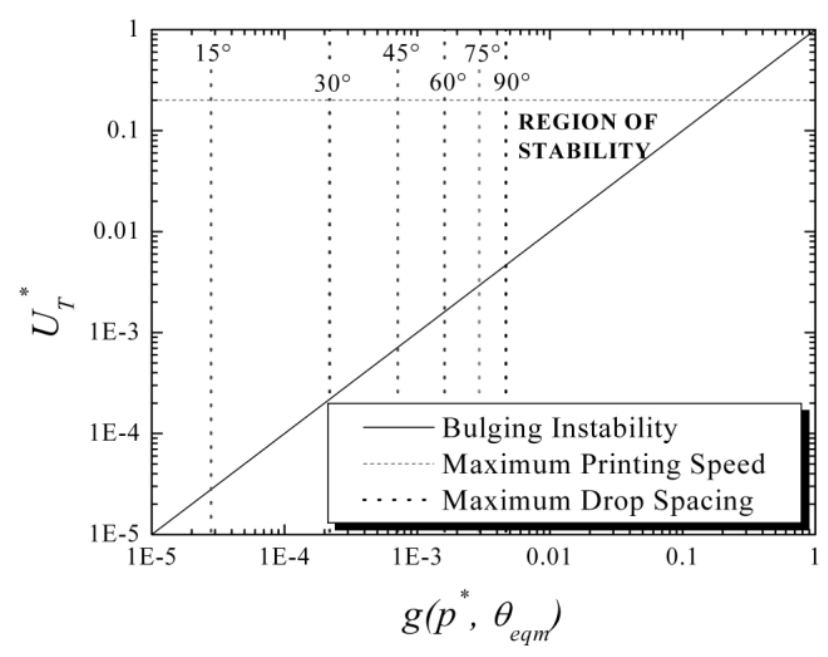

Figure 7 Graphical representation of the range of conditions (drop spacing and printer speed) under which stable parallel lines can be printed from a train of discrete drops. The diagonal line represents the onset of the bulging instability and a minimum drop spacing. The vertical dashed line represents the maximum drop spacing to forma parallel sided track. The horizontal dotted line indicates an upper limit for printer speed that represents the mechanical limitations of a given printer system and the value shown in this figure is purely arbitrary. Reproduced with permission from ref. [32]. 